\title{
How to boost the ranking of your university using self-citations? An example of the weaknesses of university ranking systems
}

\author{
Maxime Mussard ${ }^{1}$, Alex Pappachen James ${ }^{1}$ \\ ${ }^{1}$ School of Engineering, Nazarbayev University
}

The University Vel Tech, Chennai, India, is ranked 43rd in Asia according to the Times Higher Education (THE) University ranking 2016 and ranked it at 74 in THE Young University ranking 2017.

Citations weights $30 \%$ of the overall score, and it appears that Vel Tech reaches the maximum score on this criterion (100). It is ranked 1st in Asia on this criterion (the 2nd, King Abdulaziz University, got 93.3).

Although Vel Tech is not ranked in the THE world ranking, we notice that King Abdulaziz University got the same score in this ranking in Asia and worldwide. It can be extrapolated that Vel Tech is, according to this ranking, 1st or 2nd in the world on the Citations criterion (together with St George's the University of London, and followed by Stanford and MIT at 99.9).

Knowing the grades and the weighting for the other parameters of Vel Tech's ranking, the citation score is contributing for $74 \%$ of the overall performance of the University according to THE ranking ${ }^{1}$.

On further inspection from the data on SciVal, from 2012 to 2017, the Vel Tech University got 15058 citations, with a field-weighted citation impact (FWCI) of 3.18. For comparison, the FWCI of Caltech is 2.31, 2.37 for Harvard or 2.50 for the MIT $^{2}$.

Interestingly, by analysing the SciVal profile of Sundarapandian Vaidyanathan, a professor at Vel Tech, who is listed as the top author in Vel Tech University, we observe 12933 citations. Only 1\% of his papers have been published in top $10 \%$ journals by SNIP. However, $59.3 \%$ of his publications are in the top $10 \%$ most cited worldwide. He has been writing under Vel Tech affiliation since 2010. Close to $86 \%$ of the citations of the university since 2012 are coming from this professor. Scopus lists more citations (15 485), probably due to a difference in the update. Out of this citations, 14555 are self-citations, and 930 from other researchers. Notice that his H-index is 86, but drops down to 14 by removing self-citations. According to these data, about $94 \%$ of his citations are self-citations.

By considering only articles from 2012 to 2016, 13018 citations are left (very close to SciVal data), out of which 12487 are self-citations, so a ratio close to $96 \%$. By assuming the same ratio for the data from SciVal, and assuming steady ratios between the ranking and the recuperation of data, we can summarise that $(+/-1 \%)$ :

- $74 \%$ of the weight of the overall score of Vel Tech University is coming from its Citations score

- $86 \%$ of these citations are coming from Professor Sundarapandian Vaidyanathan

- $96 \%$ of his citations are self-citations 
By simply multiplying these factors, we estimate that about $83 \%$ of the citations of the university are self-citations from Professor Vaidyanathan. As we do not have access to the detailed methodology of the ranking, it's hard to find the formula relating citations and citations score. By assuming linearity, roughly $61 \%$ of the overall score of Vel Tech would be due to these self-citations. Whatever the formula is, the self-citations of Professor Vaidyanathan seem to have a tremendous influence on the ranking of Vel Tech.

This is not the first time that the THE ranking is questioned. In 2010, the ranking of the University of Alexandria was strongly influenced by the self-citations of one single author, El Naschie ${ }^{3}$. That author was previously involved in serious academic misconduct issues ${ }^{4}$. Following this scandal, the THE ranking did not improve and let the weakness of this parameter easily exploitable.

Without questioning the quality of the work of Prof. Vaidyanathan, this example shows that it is now possible for a single academic to drastically increase the ranking of its university by abusing one of the weaknesses of a given ranking system. Due to the importance of these rankings nowadays in the media or public opinion, it is time to design a resilient and reliable ranking which can annihilate future potential manipulations and pressures from university administrations or governments. Indeed, we can easily imagine a university pushing professors to self-cite their work to increase the global ranking, and thus affecting universities and academics trying to do their job honestly. Other parameters may be as well easy to abuse to boost significantly a ranking artificially (for example: parameters involving ratios of staff and/or students). Such manipulations would be very detrimental to the quality of research and teaching worldwide in the future.

It is time for the university ranking organisations to have a serious self-critic on their weaknesses and to think together with the academics about a ranking system which could be robust enough to annihilate such manipulations. Ideally, the ranking should push universities to increase the quality of their research and teaching, instead of encouraging academic misconduct. The credibility and the reputation of the academic world are now on the line.

\section{References}

[1] Times Higher Education (THE). (2017). Veltech University. [online] Available at: https://www.timeshighereducation.com/world-university-rankings/veltech-university\#ranking-dataset/608682 [Accessed 25 Jul. 2017]

[2] Scival.com. (2017). SciVal - Welcome to SciVal. [online] Available at:

http://www.scival.com/overview/summary?uri=Institution/706989 [Accessed 25 Jul. 2017].

[3] Aspenia online. (2017). International university rankings: science or quackery? [online] Available at: https://www.aspeninstitute.it/aspenia-online/article/international-university-rankings-science-or-quackery [Accessed 25 Jul. 2017].

[4] Schiermeier, Q. (2008). Self-publishing editor set to retire. Nature, 456(7221), pp.432-432. 